\title{
EDITORIAL
}

For reprint orders, please contact: reprints@futuremedicine.com

\section{Managing synchronous rectal cancer and liver metastases}
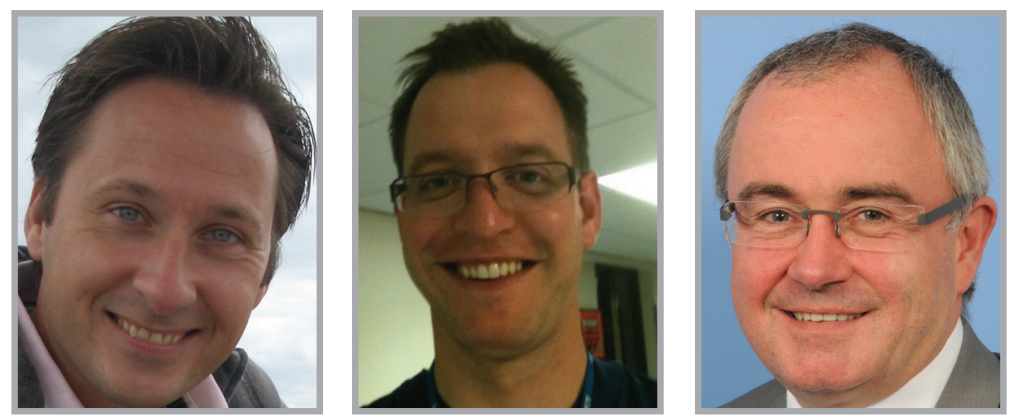

Koert Kuhlmann*,1, Simon G Fisher ${ }^{1} \&$ Graeme Poston ${ }^{1}$

At presentation, $20 \%$ of patients with colorectal cancer have liver metastases [1]. Increasingly effective chemotherapy regimens and greater options for resection have improved survival. As a result, 5-year survival can reach $55 \%$ after radical resection of all tumor sites [2]. Historically, the management strategy of colon carcinoma with liver metastases was resection of the primary tumor followed by treatment of the liver metastases. Now, despite a lack of data, a liver-first approach with synchronous colon carcinoma has been widely adopted because it results in more patients managing to complete the full treatment protocol [3].

There remains discussion regarding the treatment of patients with rectal carcinoma and synchronous (potentially) resectable liver metastases. Treatment options for these patients are systemic therapy, shortcourse radiotherapy, long-course chemoradiotherapy and surgery of the primary tumor and metastases. The principal treatment goal should be radical resection of all lesions, but the optimal sequence and choice of the different treatment options remain unclear. Furthermore, when assessing management protocols, the risk of local or distant disease progression must be considered as well as disease-free and overall survival outcomes.

\section{Symptomatic rectal tumor}

Patients with obstructive symptoms and radiographic evidence of an obstructing rectal mass should undergo decompression with a colostomy as their first step in management. The over-riding concern in such scenarios is the development of complete obstruction secondary to disease progression or tissue edema and swelling due to neoadjuvant chemoradiotherapy. An emergency colostomy would cause an interruption of therapy, result in additional recovery time and interfere with the oncological benefit of the treatment.

Many surgeons managing an endoscopically obstructing, symptomatic rectal tumor opt to create a diverting colostomy. However, a patient passing gas with liquid stools can be safely treated most of the times with only dietary modification prior to starting local radiotherapy. Although there are no randomized trials in support of this, a cohort study by Patel et al. shows that only $4.3 \%$ of patients with an

'Liver Surgery Unit, Aintree University Hospital, Lower Lane, Liverpool, L9 7AL, UK

*Author for correspondence: Tel.: +44 151525 5890; Fax: +44 151529 8547; kfdkuhlmann@yahoo.com

Future
Medicine
part of 
"The use of short-course rectal downstaging radiotherapy facilitates good control over the colorectal metastasis, again enabling completion of full treatment protocols." endoscopic obstruction progressed to a clinical obstruction needing an emergency colostomy [4].

\section{Local control of the rectal tumor}

Most patients with stage IV rectal carcinoma will have an advanced rectal tumor that should be treated locally with long-course chemoradiotherapy. This regimen combines 25 sessions of radiotherapy with a radiosensitizer, such as 5-fluorouracil [5]. In this setting, 5-fluorouracil being only a radiosensitizer has minimal systemic effects. Therefore, in this regimen the liver metastases remain untreated for a substantial period of time. If this chemoradiotherapy is then followed by resection of the primary rectal tumor, rather than systemic chemotherapy, the time interval is even be longer. Especially should postoperative complications occur after the initial rectal surgery.

An alternative local treatment option for advanced rectal tumors is short-course radiotherapy $(5 \times 5$ Gy during week 1$)$ followed by delayed rectal surgery [6]. This regimen can result in radiological tumor regression in $74 \%$ of patients and has a low toxicity profile [6]. Using this regimen for local control, the patient could start with systemic chemotherapy in week 2 avoiding the long interval without systemic treatment.

A third option is treatment with chemotherapy only. A case series of 22 rectal cancer patients showed an objective pathological response in 12 patients, including one patient with a complete response [7].

\section{Short-course chemotherapy followed by} systemic treatment \& subsequent surgery

To date, the only trial investigating short-course radiotherapy followed by systemic treatment and subsequent resection of the primary and metastases was performed by Van Dijk et al. [8]. In this Phase II trial, patients were enrolled with primary rectal cancer with liver and/or lung metastases. They received radiotherapy $(5 \times 5$ Gy) followed by capcitabin, oxaliplatin and bevacizumab for up to six cycles. Surgery was carried out $6-8$ weeks after the last bevacizumab dose. Radical surgical treatment at all sites (R0) was achieved in 36 $(72 \%)$ of the included 50 patients. Progression of the rectal tumor was not observed during the interval between radiation therapy and surgery (median: 180 days; range: 132-360). A pathologic complete response of the primary tumor was seen in $26 \%$ of patients and a pathologic near complete response in $16 \%$ of patients. These response frequencies are comparable or better than those with other neoadjuvant chemoradiation schemes [9]. There were 23 (64\%) patients who had recurrent disease within 2 years after radical surgical treatment. Only two patients had a local pelvic relapse.

\section{Liver first approach}

A liver first approach is feasible in patients with stage IV rectal carcinoma. It can avoid treatment delays caused by complications of rectal surgery. Most studies investigate combined groups of colon cancer with rectal cancer. A systematic review by Jegatheeswaran et al. [3] in 2013 found four studies of which one assessed rectal cancer patients only. Of the 121 patients included in the systemic review, 90 (74\%) completed the specified treatment protocol. Disease progression during the protocol period occurred in 23 patients (19\%).

Verhoef et al. described 23 patients with locally advanced rectal cancer and liver metastases [10]. One patient had liver resection without neoadjuvant chemotherapy followed by chemoradiotherapy. All remaining 22 patients underwent initial laparotomy after chemotherapy. Sixteen patients $(73 \%)$ completed the full treatment protocol and all were alive after a median period of 19 months. The same institute recently described their long-term results, showing a 5-year overall survival of $67 \%$ and a median progressionfree survival of 19 months for the patients that completed the full protocol [11].

A multicenter international cohort study analyzed 1004 patient with colorectal cancer and synchronous liver metastases [12]. In this study, 276 patients had a rectal primary. Although most patients underwent a primary first regimen, 15 patients underwent a liver first and 81 patients a synchronous resection. Outcome of these patients was comparable to the patients undergoing a staged resection.

\section{Need for resection of primary in irresectable liver metastases}

The primary goal of treatment in the case of unresectable liver metastases is palliation. If there is no evidence of obstruction nor tumor bleeding, there is no indication for resection of the primary [13]. Meta-analysis by Stillwell et al. found that only $18 \%$ of patients with stage IV colorectal cancer and unresectable liver metastases need surgery for their primary [14]. Unnecessary resection should be avoided as complications will 
delay systemic treatment and increase morbidity and mortality, which is significantly higher with stage IV rectal cancer resections [15].

\section{Three clinical scenarios}

\section{- Patient 1: female, age 76 years,}

\section{hypertension}

Presents with an asymptomatic T3N1 midrectal adenocarcinoma with six small bilobar liver metastases and two indeterminate lung nodules.

The patient started with short-course radiotherapy of the rectum followed by six cycles of oxaliplatin, capcitabin and bevacizumab. Bevacizumab was not added to the sixth cycle to prevent drug-related complications. Restaging showed a partial response of the rectum and stable liver metastases. 4 weeks after chemotherapy she underwent a combined resection and microwave ablation of the liver metastases. 5 weeks thereafter a laparoscopic low anterior resection was performed with a defunctioning loop ileostomy that was reversed 3 months later. The lung nodules were followed up.

\section{- Patient 2: male, age 41 years, no comorbidities}

Presents with large obstructive T4N2 low rectal carcinoma with involvement of the prostate and multiple bilobar liver metastases. The referring center performed emergency loop colostomy and started palliative chemotherapy. Patient was referred because of a partial response of the liver metastases after three cycles of chemotherapy.

Reassessment showed multiple liver metastases, possibly suitable for two-stage hepatectomy. Patient started with short-course radiotherapy followed by another three cycles of chemotherapy. Radiological restaging showed further response of the liver and stable disease of the rectum. Surgical clearance of the left liver and embolization of the right portal vein was performed 6 weeks after chemotherapy. Subsequent right hepatectomy was done 6 weeks later. Restaging of the rectum showed a partial response and open abdomino-perineal resection with partial prostatectomy was performed.

\section{- Patient 3: female, age 56 years, no comorbidities}

Presents with asymptomatic T3N1 mid rectal tumor with two liver metastases in the left lateral segment of the liver.

Patient initially started with short-course radiotherapy followed by six courses of systemic treatment. Restaging shows stable disease of liver and rectum. 6 weeks thereafter patients underwent a synchronous laparoscopic metastasectomy of the two liver metastases and a laparoscopic low anterior resection with defunctioning loop ileostomy which was reversed 10 weeks later.

\section{Conclusion}

In conclusion, treatment of stage IV rectal cancer is complex and supporting evidence is scarce. There is growing evidence to support the liver first strategy in stage IV rectal cancer. It enables more patients to complete full treatment protocols. The use of short-course rectal downstaging radiotherapy facilitates good control over the colorectal metastasis, again enabling completion of full treatment protocols. Synchronous resection forms part of the multimodal treatment of stage IV colon cancer. Optimal treatment requires good coordination of the many specialties involved and is best managed by a regional Multidisciplinary Team Meeting whose membership is composed of the responsible clinicians from each specialty $[16,17]$.

\section{Financial \& competing interests disclosure}

$G$ Poston has the following to disclose. Advisory board member of: Merck Serono, Sanofi Aventis, Novartis, Ipsen, BTG Biocompatibles. He is a Bayer speaker panel member of: Merck Serono, BTG Biocompatibles and Ipsen. The authors have no other relevant affiliations or financial involvement with any organization or entity with a financial interest in or financial conflict with the subject matter or materials discussed in the manuscript apart from those disclosed.

No writing assistance was utilized in the production of this manuscript.

\section{References}

1 Leporrier J, Maurel J, Chiche L, Bara S, Segol P, Launoy G. A population-based study of the incidence, management and prognosis of hepatic metastases from colorectal cancer. Br. J. Surg. 93, 465-474 (2006).
2 Kopetz S, Chang GJ, Overman MJ et al. Improved survival in metastatic colorectal cancer is associated with adoption of hepatic resection and improved chemotherapy. J. Clin. Oncol. 27, 3677-3683 (2009).

3 Jegatheeswaran S, Mason JM, Hancock HC, Siriwardena AK. The liver-first approach to the management of colorectal cancer with synchronous hepatic metastases: a systematic review. JAMA Surg. 148, 385-391 (2013).

4 Patel JA, Fleshman JW, Hunt SR et al. Is an elective diverting colostomy warranted in patients with an endoscopically obstructing rectal cancer before neoadjuvant 
chemotherapy? Dis. Colon Rectum 55(3), 249-255 (2012).

5 Gérard J-P, Conroy T, Bonnetain F et al. Preoperative radiotherapy with or without concurrent fluorouracil and leucovorin in T3-4 rectal cancers: results of FFCD 9203. J. Clin. Oncol. 24, 4620-4625 (2006).

6 Pettersson D, Holm T, Iversen H, Blomqvist L, Glimelius B, Martling A. Preoperative short-course radiotherapy with delayed surgery in primary rectal cancer. Br. J. Surg. 99, 577-583 (2012).

7 Bensignor T, Brouquet A, Dariane C et al. Pathologic response of locally advanced rectal cancer to preoperative chemotherapy without pelvic irradiation. Colorectal Dis. doi:10.1111/ codi.12879 (2015) (Epub ahead of print).

8 Van Dijk TH, Tamas K, Beukema JC et al. Evaluation of short-course radiotherapy followed by neoadjuvant bevacizumab, capecitabine, and oxaliplatin and subsequent radical surgical treatment in primary stage IV rectal cancer. Ann. Oncol. 24, 1762-1769 (2013).
9 Resch G, De Vries A, Öfner D et al. Preoperative treatment with capecitabine, bevacizumab and radiotherapy for primary locally advanced rectal cancer - a two stage Phase II clinical trial. Radiother. Oncol. 102, 10-13 (2012).

10 Verhoef C, van der Pool AEM, Nuyttens JJ, Planting AST, Eggermont AMM, de Wilt JHW. The 'liver-first approach' for patients with locally advanced rectal cancer and synchronous liver metastases. Dis. Colon Rectum 52, 23-30 (2009).

11 Ayez N, Burger JW a, van der Pool AE et al. Long-term results of the 'liver first' approach in patients with locally advanced rectal cancer and synchronous liver metastases. Dis. Colon Rectum 56, 281-287 (2013).

12 Mayo SC, Pulitano C, Marques $\mathrm{H}$ et al. Surgical management of patients with synchronous colorectal liver metastasis: a multicenter international analysis. J. Am. Coll. Surg. 216, 707-716 (2013).

13 Damjanov N, Weiss J, Haller DG. Resection of the primary colorectal cancer is not necessary in nonobstructed patients with metastatic disease. Oncologist 14, 963-969 (2009).

14 Stillwell AP, Ho YH, Veitch C. Systematic review of prognostic factors related to overall survival in patients with stage IV colorectal cancer and unresectable metastases. World J. Surg. 35, 684-692 (2011).

15 Evans MD, Escofet X, Karandikar SS, Stamatakis JD. Outcomes of resection and non-resection strategies in management of patients with advanced colorectal cancer. World J. Surg. Oncol. 7, 28 (2009).

16 Borras JM, Albreht T, Audisio R et al. Policy statement on multidisciplinary cancer care. Eur. J. Cancer 50, 475-480 (2014).

17 Jones RP, Vauthey J-N, Adam R et al. Effect of specialist decision-making on treatment strategies for colorectal liver metastases. $\mathrm{Br}$. J. Surg. 99, 1263-1269 (2012). 